\title{
Downflows and structure above LDE arcades: Possible signatures of reconnection?
}

\author{
D. E. McKenzie ${ }^{1}$ and H. S. Hudson ${ }^{2}$ \\ ${ }^{1}$ Montana State University, Bozeman, Montana, U.S.A. \\ ${ }^{2}$ Solar Physics Research Corporation, Tucson, Arizona, U.S.A. \\ (Received May 11, 2000; Revised September 12, 2000; Accepted October 5, 2000)
}

\begin{abstract}
We will introduce a set of observations made during the decay phase of long duration event (LDE) flares on the Sun. In a number of events, the soft X-ray images from Yohkoh SXT indicate a downward directed flow field in the region immediately above the flare arcade. These are tentatively identified as evidence of reconnection outflows. The prototypical event is the M5 flare of 20 January 1999, presented by McKenzie and Hudson (1999); since the time that paper was completed, sixteen more events have been found in the interval April 1998 to February 2000. As in the 20 January event, the speeds of downflow are $35-500 \mathrm{~km} \mathrm{~s}^{-1}$, lower than both the freefall speed and the typically assumed Alfvén speed. The motion is evidenced by both dark and bright (i.e., X-ray emitting) features, some of which may have a looplike morphology; no cool counterparts have been detected in H $\alpha$ or EUV observations. Movies depicting some of the LDE downflows were presented at this meeting; these also appear in the journal Solar Physics (McKenzie, 2000), since they cannot be presented in these Proceedings.
\end{abstract}

\section{Introduction}

McKenzie and Hudson (1999) described downward directed plasma flows above a long-duration solar flare arcade, and offered some interpretations. The images in Fig. 1 are excerpts from the Yohkoh Soft X-ray Telescope (SXT) movie of this event, and show the appearance of the arcade and the fan of bright (in X-rays) rays above the arcade. (The papers by McKenzie and Hudson (1999) and McKenzie (2000) contain images which emphasize the downflowing motion in the 20 January 1999 flare, and so we will not reproduce them here.) Since the time of that report, many more solar flare events with the same kind of "supra-arcade downflow" velocity fields have been discovered. We present here a list of the flare events, and a summary of the characteristics of the flows. We consider the interpretation that the velocity fields detected by Yohkoh SXT are evidence of reconnection outflow above the solar flare arcades.

\section{List of Supra-Arcade Downflow Flares}

McKenzie and Hudson (1999) described a solar flare which occurred on 20 January 1999; it was remarkable primarily because of the plasma velocity field observed above the long-duration event (LDE) arcade. The velocity field was evidenced by the motion of X-ray dark features, traveling downward into the top of the arcade. It has since been found that the 20 January 1999 event is not the only flare which shows this supra-arcade downflow; rather, the 20 January 1999 event is the prototype of a growing set which now includes 17 solar flares between April 1998 and February 2000. Table 1 summarizes these flares, giving (i) the UT times of flare initiation and peak, as measured by the soft X-

Copy right(c) The Society of Geomagnetism and Earth, Planetary and Space Sciences (SGEPSS); The Seismological Society of Japan; The Volcanological Society of Japan; The Geodetic Society of Japan; The Japanese Society for Planetary Sciences. ray flux detected by the GOES spacecraft and reported by the NOAA Space Environment Center, (ii) the magnitude of the flare, in GOES classification, (iii) the UT time-span during which Yohkoh SXT recorded downward-directed flows, and (iv) the plane-of-sky speeds of the downward flow, measured by tracking distinct features in successive SXT images.

All the X-ray flares listed in Table 1 were associated with coronal mass ejections (CMEs). This was confirmed by the online observing records of SOHO/LASCO and Mauna Loa Solar Observatory. The speeds of observed motion range from 35 to $500 \mathrm{~km} \mathrm{~s}^{-1}$, below both the gravitational freefall speed $\left(620 \mathrm{~km} \mathrm{~s}^{-1}\right)$ and the commonly assumed value of the Alfvén speed $\left(1000 \mathrm{~km} \mathrm{~s}^{-1}\right)$ in the solar corona. As will be mentioned below, there does not seem to be any direct evidence for free-falling blobs of cool material.

In five cases (09 May 1998, 16 August 1998, 25 July 1999, 28 November 1999, and 22 February 2000), we observe $X$ ray emitting features in downward motion, not just the $\mathrm{X}$-ray voids of McKenzie and Hudson (1999). Figure 2 shows some frames excerpted from the 16 August 1998 movie; the position of a shrinking X-ray emitting feature is indicated by arrows in that figure. The $\mathrm{X}$-ray voids typically have diameters on the order of $10^{4} \mathrm{~km}$; the X-ray emitting features are of a similar diameter and give the impression of a looplike morphology. In all the flares listed in Table 1, fans of bright "rays" like those of Švestka et al. (1998) and McKenzie and Hudson (1999) are observed atop the arcades (e.g., see Fig. 1).

\section{Interpretations}

The initial report by McKenzie and Hudson (1999) offered three interpretations which included (a) coronal rain-like material falling back from the CME, (b) large-scale magnetic islands within the current sheet, and (c) reconnected flux tubes linking through the current sheet. The work reported 


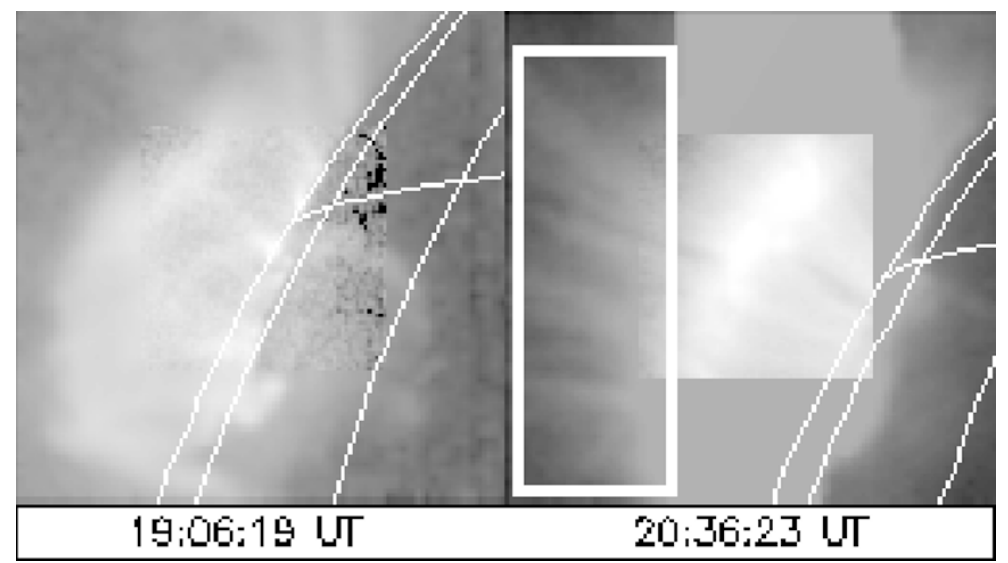

Fig. 1. Excerpts from SXT movie of 20 January 1999 flare event, described by McKenzie and Hudson (1999). The lefthand frame shows the appearance of the arcade early in the flare: the arcade is easily discernible, but the fan above the arcade has not yet developed. The righthand frame shows the appearance of the arcade and the fan of rays above the arcade during the decay phase of the flare. Motion was detected in the region indicated by the box. A heliographic grid is superimposed in both frames to show the location of the solar limb; the field of view is 5.2 arcminutes ( 225 megameters) square. The middle part of the top of the righthand frame is obscured by CCD pixel saturation.

Table 1. List of known supra-arcade downflow events, as of the time of this presentation. X-ray flare classifications and start- and peak-times are from GOES spacecraft, as reported by the NOAA Space Environment Center. All these events were associated with coronal mass ejections. Speeds have not been measured for all the detected flows, but the values here are representative of the range of observed speeds.

\begin{tabular}{lcccr}
\hline \multicolumn{1}{c}{ Date } & $\begin{array}{c}\text { GOES start, peak } \\
(\mathrm{UT})\end{array}$ & $\begin{array}{c}\text { GOES } \\
\text { classification }\end{array}$ & $\begin{array}{c}\text { SXT flows } \\
\text { observed (UT) }\end{array}$ & $\begin{array}{r}\text { Speeds } \\
\left(\mathrm{km} \mathrm{s}^{-1}\right)\end{array}$ \\
\hline 20 Apr. 1998 & $09: 10,10: 21$ & M1.4 & $09: 43-16: 42$ & $45-130$ \\
23 Apr. 1998 & $05: 35,05: 55$ & X1.2 & $06: 55-07: 12$ & $200-400$ \\
27 Apr. 1998 & $08: 55,09: 20$ & X1.0 & $09: 38-10: 36$ & \\
09 May 1998 & $03: 04,03: 40$ & M7.7 & $04: 59-05: 45$ & \\
16 Aug. 1998 & $17: 37,18: 21$ & M3.1 & $18: 27-19: 14$ & \\
18 Aug. 1998 & $22: 10,22: 19$ & X4.9 & $23: 50-00: 26$ & \\
30 Sep. 1998 & $13: 08,13: 50$ & M2.8 & $13: 32-14: 07$ & \\
23 Nov. 1998 & $10: 59,11: 21$ & M3.1 & $11: 52-12: 34$ & $140-300$ \\
18 Dec. 1998 & $17: 13,17: 22$ & M8.0 & $18: 18-18: 23$ & \\
20 Jan. 1999 & $19: 06,20: 04$ & M5.2 & $20: 36-21: 32$ & $90-500$ \\
03 May 1999 & $05: 36,06: 02$ & M4.4 & $06: 04-07: 08$ & $180-300$ \\
08 May 1999 & $10: 36,10: 58$ & M1.6 & $11: 14-11: 24$ & \\
08 May 1999 & $14: 22,14: 40$ & M4.6 & $14: 33-14: 52$ & \\
11 May 1999 & $21: 43,22: 08$ & C4.7 & $21: 59-22: 02$ & \\
25 Jul. 1999 & $13: 08,13: 38$ & M2.4 & $13: 33-14: 00$ & \\
28 Nov. 1999 & $18: 00,19: 17$ & M1.6 & $21: 33-22: 16$ & $34-100$ \\
22 Feb. 2000 & $19: 54,21: 47$ & M1.1 & $20: 28-21: 23$ & 150 \\
\hline
\end{tabular}

in this presentation is intended to explore the first and third possibilities; these are more easily tested with the currently available data. This presentation is by necessity brief, given the nature of this meeting. A more in-depth examination of all the available data for twelve of these flares can be found in McKenzie (2000).

The Yohkoh SXT is a broadband instrument, sensitive to emission from plasmas with temperatures between 2 and 20 million kelvins (Tsuneta et al., 1991). We have compared the SXT imagery for some of these 17 flare events with the available extreme ultraviolet (EUV) and hydrogen Balmer alpha $(\mathrm{H} \alpha)$ solar imagery for the same events, with the expectation that if the X-ray voids described by McKenzie and Hudson (1999) correspond to free-falling blobs of CME material, these blobs might be emitting radiation and thus be visible in solar imagery of some passband other than the soft $\mathrm{X}$-rays visible to SXT. Solar plasma with temperature near 1.5 million kelvins will emit radiation with wavelength 195 $\AA$ A; plasma near 10 thousand kelvins will emit $\mathrm{H} \alpha$ radiation. For several of the flares in Table 1, EUV (from SOHO/EIT, Delaboudinière et al., 1995) and $\mathrm{H} \alpha$ (from Mauna Loa Solar Observatory) images were retrieved and analyzed. The 


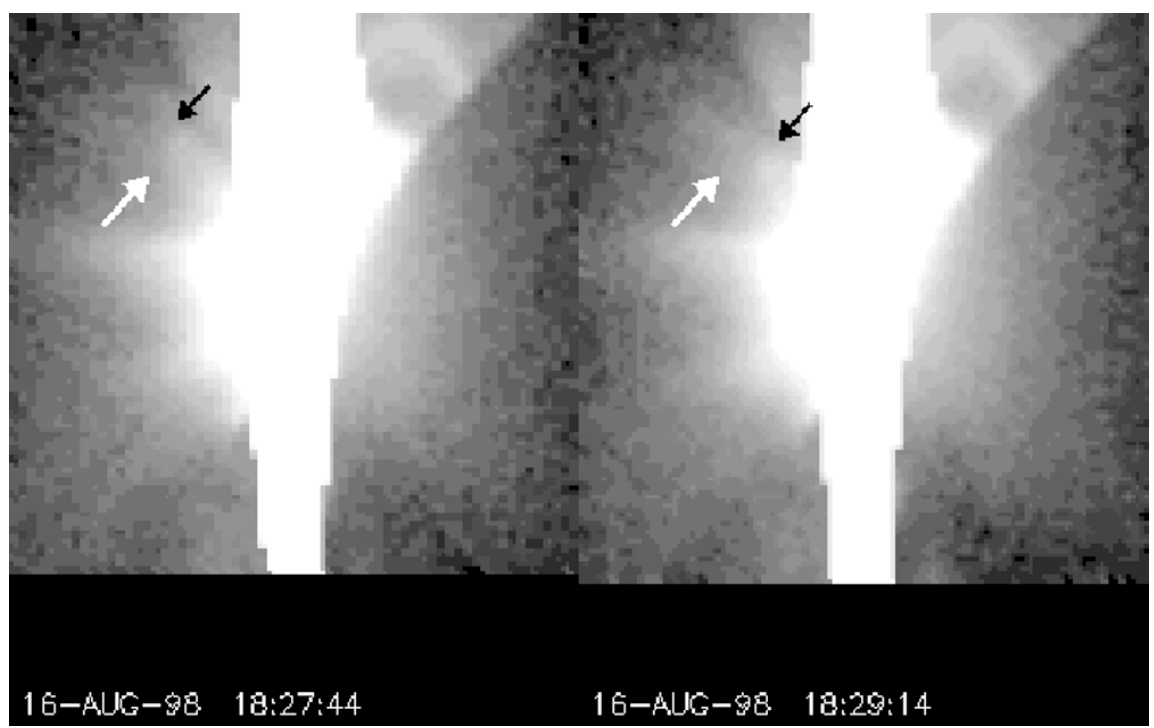

Fig. 2. Excerpts from 16 August 1998 SXT movie. The black arrow indicates the apex of an X-ray emitting looplike feature, observed to shrink into the top of the arcade; the white arrow is at a fixed position, for comparison. The field of view is 5.2 arcminutes (225 megameters) square. The middle part of the arcade is obscured in these images by CCD pixel saturation.

EUV data show the flare arcades clearly, but do not reveal the arcade fans or the motions above the arcades. Similarly, the $\mathrm{H} \alpha$ data reveal the post-flare loops commonly observed in events of this sort, but do not show any evidence of structures or motion above the arcades. For a fuller description, including a case-by-case synopsis of the data for 12 of the flares, the reader is encouraged to examine McKenzie (2000).

The non-detections in EUV and $\mathrm{H} \alpha$ imagery suggest that the interpretation in terms of CME material falling back under the force of gravity is at best incomplete. On the other hand, the X-ray data from Yohkoh SXT show not only the Xray voids as described by McKenzie and Hudson (1999), but also several cases of bright, X-ray emitting features moving downwards into the top of the arcade. These X-ray emitting features cannot be interpreted as material "falling back" from the CME. That the X-ray emitting features are vaguely looplike in appearance strongly suggests that the interpretation in terms of magnetic field line shrinkage (i.e., reconnection outflow) is more nearly correct.

We suggest that the observed X-ray voids and shrinking $\mathrm{X}$-ray emitting features might be simultaneously described by a common cartoon. In Fig. 3, we sketch a magnetic flux tube (bundle of field lines) which has reconnected high above the post-eruption arcade. This magnetic flux tube, linking through the supra-arcade fan/sheet, retracts downward in response to the force of magnetic tension. If the flux tube has filled with plasma (e.g., via ablation of chromospheric plasma (Forbes and Malherbe, 1986; Schmieder et al., 1987; Švestka, 1989)), then the appearance should be similar to the looplike, X-ray emitting features observed in five of the 17 events. However, if the flux tube has not yet filled with plasma for some reason, then the appearance in X-ray images is expected to be very similar to that of the X-ray voids: the $\mathrm{X}$-ray voids themselves would represent the cross-section of the flux tube linking through the supra-arcade sheet.

These observations, if interpreted as magnetic field line shrinkage, are different from, and considerably more direct

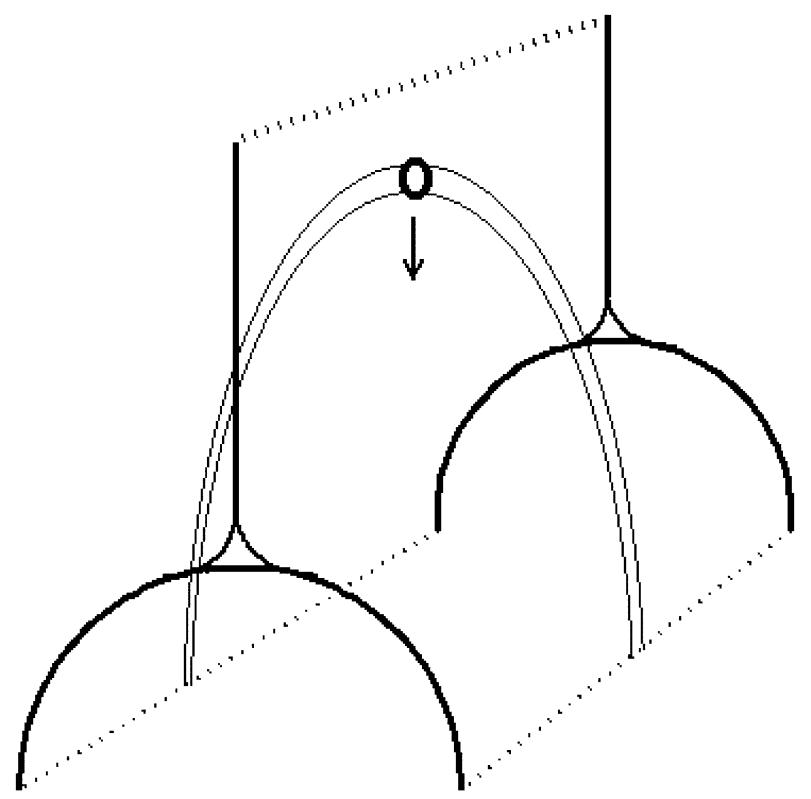

Fig. 3. Cartoon interpretation of the supra-arcade downflows. The X-ray emitting features and the X-ray voids are both interpreted as reconnected flux tubes shrinking under magnetic tension.

than, those of Hiei and Hundhausen (1996) and Forbes and Acton (1996). Those authors described motions which could be construed as the retraction of magnetic field lines from reconnection sites. Those motions, however, were 10 to 100 times slower than the current observations, which are slower again than what is commonly expected for reconnection outflow. The outflow speed is ideally calculated to be equal to the upstream Alfvén speed, and thus entirely determined by the conditions in the region of inflow. Forbes and Acton (1996) remind us that the outflow speed is also affected by the plasma pressure downstream, and thus may be significantly lower than the upstream Alfvén speed. 


\section{Open Questions}

The discovery by Yokoyama et al. (2001) of likely inflow to a post-eruption reconnection site, coupled with the observations of supra-arcade downflows, interpretable as reconnection outflows, suggest that some of the heretofore unobserved reconnection-associated velocity fields are now within the grasp of solar observational methods. These new observations of flow fields may provide new quantitative tools with which to understand the reconnection process and its environment in solar flares.

The speeds of supra-arcade downflow (35-500 $\mathrm{km} \mathrm{s}^{-1}$ ) are slower than the typically assumed Alfvén speed (1000 $\mathrm{km} \mathrm{s}^{-1}$ ). This may not be a serious problem, because as Forbes and Acton (1996) point out, the outflow speed is affected by the conditions in the outflow region. Additionally, the Alfvén speed in the inflow region is not well known, due to the difficulty in measuring the physical conditions in the corona. But the situation needs to be explored fully, because we might be able to glean some useful information from measurements of the outflow speed, e.g., implications for the plasma beta in the region.

The fan of rays on top of the arcades is still not well understood. The suggestion by Švestka et al. (1998) that the rays might represent outward-flowing (i.e., away from the Sun) plasma jets has not been supported by direct evidence of motion in that direction. Data from Yohkoh SXT and SOHO/EIT indicate that the temperatures of these features may exceed 8-10 MK; the rays are seen to persist for several hours after the beginning of the flare. The possibility that the rays result from an interchange instability - possibly a "fluting" aligned with the magnetic field outside the current sheet-is being considered, but at the time of this presentation such an interpretation remains speculative.

\section{References}

Delaboudinière, J.-P., et al., EIT: Extreme-ultraviolet imaging telescope for the SOHO mission, Sol. Phys., 162, 291, 1995.

Forbes, T. G. and L. W. Acton, Reconnection and field line shrinkage in solar flares, ApJ, 459, 330, 1996.

Forbes, T. G. and J. M. Malherbe, A shock condensation mechanism for loop prominences, ApJ, 302, L67, 1986.

Hiei, E. and A. J. Hundhausen, Development of a coronal helmet streamer of 24 January 1992, in Magnetodynamic Phenomena in the Solar Atmosphere, edited by Y. Uchida, T. Kosugi, and H. Hudson, p. 125, Kluwer Academic Publishers, Dordrecht, Holland, 1996.

McKenzie, D. E., Supra-arcade downflows in long-duration solar flare events, Sol. Phys., 195, 381, 2000.

McKenzie, D. E. and H. S. Hudson, X-ray observations of motions and structure above a solar flare arcade, ApJ, 519, L93, 1999.

Schmieder, B., T. G. Forbes, J. M. Malherbe, and M. E. Machado, Evidence for gentle chromospheric evaporation during the gradual phase of large solar flares, ApJ, 317, 956, 1987.

Švestka, Z., Solar flares-the gradual phase, Sol. Phys., 121, 399, 1989.

Švestka, Z., F. Fárník, H. Hudson, and P. Hick, Large-scale active coronal phenomena in Yohkoh SXT images - IV. Solar wind streams from flaring active regions, Sol. Phys., 182, 179, 1998.

Tsuneta, S., et al., The soft X-ray telescope for the solar-A mission, Sol. Phys., 136, 37, 1991.

Yokoyama, T., et al., Clear evidence of reconnection inflow of a solar flare, ApJ, 546, L69, 2001.

D. E. McKenzie (e-mail: mckenzie@mithra.physics.montana.edu) and H. S. Hudson 